\title{
Guest Editorial: Special Issue Wireless Personal Multimedia Communications
}

\author{
Ryuji Kohno • Matti Latvaho • Neeli Rashmi Prasad • \\ António Rodrigues
}

Published online: 17 September 2010

(C) Springer Science+Business Media, LLC. 2010

Wireless communications are rapidly evolving and start to face enormous challenges to address all the users and network providers' needs in terms of available bandwidth, overall network capacity or innovative services to improve revenue.

These challenges involve the evolution of the radio interface, the introduction in the standards of cognitive radio systems, multi-user MIMO or indeed cooperation involving all of the infra structured networks also exploiting cross-layer approaches and novel developments.

The series of international symposia on Wireless Personal Multimedia Communications (WPMC) constitutes a rich technical research and business environment where people from all over the world bring their own achievements and results for fostering the progress in mobile communications, aiming for breakthrough ideas to take us well into the next decade.

This special issue was planned to include some of the best papers in a wide range of research areas timely when WPMC 2008 was held in Saariselka, Finland on September 8-11, 2008.

\footnotetext{
R. Kohno

Division of Physics, Electrical \& Computer Engineering, Yokohama National University, 79-5 Tokiwadai, Hodogaya-ku, Yokohama 240-8501, Japan

e-mail: kohno@ynu.ac.jp

M. Latvaho

Centre for Wireless Communications, University of Oulu, P.O. Box 444, 90571 Oulu, Finland

e-mail: matla@ee.oulu.fi

\section{N. R. Prasad}

Center for TeleInFrastruktur (CTIF), Aalborg University, Fredrik Bajers Vej 7, Room \#: A3-202, 9220 Aalborg East, Denmark

e-mail: np@es.aau.dk

\section{A. Rodrigues $(\varangle)$}

Instituto Superior Técnico, Departamento de Engenharia Electrotécnica e de Computadores,

Av. Rovisco Pais, 1, 1049-001 Lisboa, Portugal

e-mail: antonio.rodrigues@1x.it.pt
} 
It contains fourteen papers which were accepted after strict peer review. Twenty-five papers have been selected among the excellent papers originally presented at WPMC 2008, including awarded papers. The papers were then revised and resubmitted to this special issue.

This issue covers several different topics of research, starting with papers addressing the capacity of multi user channels and code performance over time-varying channels. Then, specific developments in the use of MIMO are presented in a set of three papers. These are followed by a number of papers that deal with cooperation, cross-layer issues and cognition and multi-hop networks. The issue is finalized with a set of papers on positioning, VoIP performance and a radio resource management scheduling scheme.

The editors would like to express their sincere thanks to all the authors that helped in the construction of this special issue, to the reviewers who provided us with their revision reports and, in particular, we would like to thank Cindy Zitter and Mark de Jongh, for their assistance in the editing of this special issue. 